\title{
Crystal structure of bis(1-pyridin-2-yl-ethylidene)-p-tolylamine)nickel dithiocyanat, $\left[\mathrm{Ni}\left(\mathrm{C}_{14} \mathrm{H}_{14} \mathbf{N}_{2}\right)_{2}\right](\mathrm{NCS})_{2}$
}

\author{
Ya-Ning Guo* \\ Baoji University of Arts and Sciences, Department of Chemistry, Baoji, 721007 Shaanxi, P. R. China
}

Received September 2, 2009, accepted and available on-line September 18, 2009; CCDC no. 1267/2757

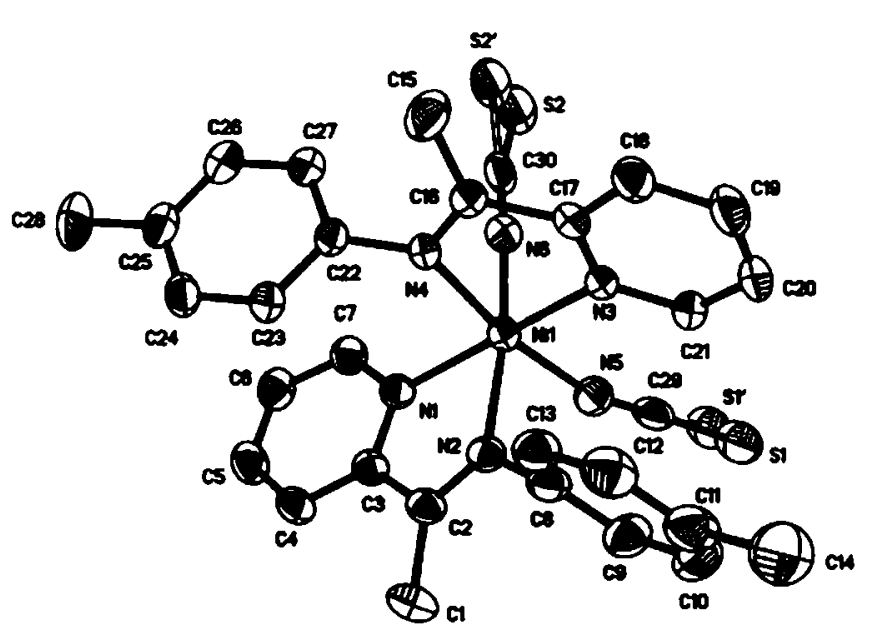

\begin{abstract}
$\mathrm{C}_{30} \mathrm{H}_{28} \mathrm{~N}_{6} \mathrm{NiS}_{2}$, triclinic, $P \overline{1}$ (no. 2), $a=9.9405(8) \AA$,

$b=11.641(1) \AA, c=14.648(2) \AA, \alpha=73.846(1)^{\circ}$,

$\beta=75.172(1)^{\circ}, \gamma=77.102(2)^{\circ}, V=1552.6 \AA^{3}, Z=2$,

$R_{g t}(F)=0.049, w R_{\text {ref }}\left(F^{2}\right)=0.113, T=298 \mathrm{~K}$.
\end{abstract}

\section{Source of material}

1-Pyridin-2-yl-ethanone $(0.1 \mathrm{mmol}, 12.1 \mathrm{mg}), \mathrm{NH}_{4} \mathrm{SCN}$ $(0.1 \mathrm{mmol}, 7.6 \mathrm{mg}), \mathrm{Ni}\left(\mathrm{CH}_{3} \mathrm{COO}\right)_{2}(0.1 \mathrm{mmol}, 17.7 \mathrm{mg})$ and $p$ tolylamine $(0.1 \mathrm{mmol}, 10.7 \mathrm{mg})$ were dissolved in methanol $(10 \mathrm{ml})$. The mixture was stirred for $30 \mathrm{~min}$ at room temperature to give a clear brown solution. After keeping this solution in air for $7 \mathrm{~d}$, brown block-shaped crystals of the title compound were obtained on slow evaporation of the solvent. The crystals were collected, washed with methanol and dried in a vacuum desiccator using anhydrous $\mathrm{CaCl}_{2}$ (yield $54 \%$ ).

\section{Discussion}

Schiff base complexes have been extensively investigated. Up to now it is still a hot research field, which could be attributed to their strong coordination ability and versatile coordination modes, antiseptic and antitumor activities $[1,2]$ together with the special catalysis behavior and magnetism properties [3]. Schiff bases of pyridone and its complexes continue to be a subject of current interest. However, only limited Schiff bases of ketone have been reported up to now $[4,5]$.

The crystal structure of the title compound is composed of a cation $\mathrm{Ni}^{2+}$, two Schiff base ligands and two SCN anion. The nickel atom is octahedrally coordinated by N1 and N2 from the Schiff base ligand, N3 and N4 from another Schiff base ligand and N5, N6 from two SCN , which is in agreement with the rule of the op- timal coordination number [6]. The bond length and bond angles of Schiff base ligands are in the normal ranges and in agreement with that of ketone Schiff base [7]. The $\mathrm{C} 2=\mathrm{N} 2$ and $\mathrm{C} 16=\mathrm{N} 4$ bond lengths of $1.281(4)$ and $1.268(4) \AA$ are conform to a double bonds. The pyridine rings and phenyl rings are nearly planar and the mean deviations of the atoms from the least-squares planes $A$ (N1/C3-C7), B (C8-C13), C (N3/C17-C21), D (C22-C27) are $0.006,0.002,0.011$ and $0.006 \AA$, respectively. The methyl groups are slightly twisted out of benzene mean plane, atom $\mathrm{C} 14$ deviates from ring B by $0.049 \AA$, whereas C28 by $0.071 \AA$. The Schiff base ligands adopt a trans configuration about the $\mathrm{C}=\mathrm{N}$ bond. $\mathrm{T}$ avoid steric conflicts, the two rigid ring systems of Schiff base ligand, pyridine ring and phenyl ring are rotated with respect to each other. Rings A, B and C, D are oriented at dihedral angles of $\angle \mathrm{A} / \mathrm{B}=88.43(1)^{\circ}$ and $\angle \mathrm{C} / \mathrm{D}=77.66(1)^{\circ}$.

Table 1. Data collection and handling.

$\begin{array}{ll}\text { Crystal: } & \text { brown block, size } 0.40 \times 0.41 \times 0.45 \mathrm{~mm} \\ \text { Wavelength: } & \text { Mo } K_{\alpha} \text { radiation }(0.71073 \AA) \\ \mu: & 7.88 \mathrm{~cm}^{-1} \\ \text { Diffractometer, scan mode: } & \text { Bruker SMART CCD, } \varphi / \omega \\ 2 \theta_{\max }: & 50.04^{\circ} \\ N(h k l)_{\text {measured, }} N(h k l)_{\text {,unique: }}: & 8083,5381 \\ \text { Criterion for } I_{\text {obs, }} N(h k l)_{\text {gt }}: & I_{\text {obs }}>2 \sigma\left(I_{\text {obs }}\right), 3369 \\ N(\text { param })_{\text {refined: }} & 364 \\ \text { Programs: } & \text { SHELXS-97 [8], SHELXL-97 [9], } \\ & \text { SHELXTL [10] }\end{array}$

Table 2. Atomic coordinates and displacement parameters (in $\AA^{2}$ ).

\begin{tabular}{llllll}
\hline Atom & Site & $x$ & $y$ & $z$ & $U_{\text {iso }}$ \\
\hline H(1A) & $2 i$ & 0.2158 & 0.6869 & 0.4634 & 0.114 \\
H(1B) & $2 i$ & 0.3163 & 0.6160 & 0.5347 & 0.114 \\
H(1C) & $2 i$ & 0.1640 & 0.5859 & 0.5539 & 0.114 \\
H(4) & $2 i$ & 0.2781 & 0.4012 & 0.6329 & 0.076 \\
H(5) & $2 i$ & 0.3495 & 0.1982 & 0.6973 & 0.088 \\
H(6) & $2 i$ & 0.4702 & 0.0742 & 0.5957 & 0.083 \\
H(7) & $2 i$ & 0.5170 & 0.1541 & 0.4307 & 0.073 \\
H(9) & $2 i$ & 0.4117 & 0.7366 & 0.2862 & 0.090 \\
H(10) & $2 i$ & 0.3313 & 0.9154 & 0.1863 & 0.106 \\
H(12) & $2 i$ & -0.0072 & 0.7861 & 0.1916 & 0.098 \\
H(13) & $2 i$ & 0.0688 & 0.6068 & 0.2932 & 0.079 \\
H(14A) & $2 i$ & 0.0791 & 0.9677 & 0.0566 & 0.198 \\
H(14B) & $2 i$ & 0.1777 & 1.0338 & 0.0854 & 0.198 \\
H(14C) & $2 i$ & 0.0219 & 1.0311 & 0.1436 & 0.198 \\
H(15A) & $2 i$ & 0.1394 & 0.2654 & 0.1281 & 0.143 \\
H(15B) & $2 i$ & 0.0167 & 0.3550 & 0.1753 & 0.143 \\
H(15C) & $2 i$ & 0.0720 & 0.2294 & 0.2391 & 0.143 \\
H(18) & $2 i$ & 0.1390 & 0.4850 & 0.0368 & 0.083 \\
H(19) & $2 i$ & 0.2312 & 0.6523 & -0.0657 & 0.087 \\
H(20) & $2 i$ & 0.4097 & 0.7155 & -0.0282 & 0.080 \\
& & & & & \\
\hline & & & & &
\end{tabular}


Table 2. Continued.

\begin{tabular}{llllll}
\hline Atom & Site & $x$ & $y$ & $z$ & $U_{\text {iso }}$ \\
\hline H(21) & $2 i$ & 0.4958 & 0.6093 & 0.1063 & 0.068 \\
H(23) & $2 i$ & 0.0947 & 0.3231 & 0.4591 & 0.067 \\
H(24) & $2 i$ & 0.0527 & 0.1658 & 0.5916 & 0.079 \\
H(26) & $2 i$ & 0.2546 & -0.0703 & 0.4311 & 0.083 \\
\hline
\end{tabular}

Table 2. Continued.

\begin{tabular}{llllll}
\hline Atom & Site & $x$ & $y$ & $z$ & $U_{\text {iso }}$ \\
\hline H(27) & $2 i$ & 0.3013 & 0.0879 & 0.2980 & 0.073 \\
H(28A) & $2 i$ & 0.1090 & -0.1402 & 0.5955 & 0.141 \\
H(28B) & $2 i$ & 0.0436 & -0.0469 & 0.6607 & 0.141 \\
H(28C) & $2 i$ & 0.2060 & -0.0961 & 0.6435 & 0.141 \\
\hline
\end{tabular}

Table 3. Atomic coordinates and displacement parameters (in $\AA^{2}$ ).

\begin{tabular}{|c|c|c|c|c|c|c|c|c|c|c|c|}
\hline Atom & Site & Occ & $x$ & $y$ & $z$ & $U_{11}$ & $U_{22}$ & $U_{33}$ & $U_{12}$ & $U_{13}$ & $U_{23}$ \\
\hline $\mathrm{Ni}(1)$ & $2 i$ & & $0.42345(5)$ & $0.39670(4)$ & $0.28585(3)$ & $0.0562(3)$ & $0.0515(3)$ & $0.0390(3)$ & $-0.0165(2)$ & $-0.0070(2)$ & $-0.0090(2)$ \\
\hline$S(1)$ & $2 i$ & $0.68(1)$ & $0.8243(6)$ & $0.6027(8)$ & $0.1623(4)$ & $0.090(2)$ & $0.124(4)$ & $0.080(2)$ & $-0.064(3)$ & $0.014(2)$ & $-0.037(3)$ \\
\hline$S(2)$ & $2 i$ & $0.30(1)$ & $0.705(1)$ & $0.0564(8)$ & $0.1730(7)$ & $0.166(4)$ & $0.058(1)$ & $0.080(1)$ & $-0.015(2)$ & $0.016(2)$ & $-0.0179(9)$ \\
\hline$S\left(1^{\prime}\right)$ & $2 i$ & 0.32 & $0.850(1)$ & $0.548(1)$ & $0.1853(8)$ & $0.090(2)$ & $0.124(4)$ & $0.080(2)$ & $-0.064(3)$ & $0.014(2)$ & $-0.037(3)$ \\
\hline$S\left(2^{\prime}\right)$ & $2 i$ & 0.70 & $0.6367(7)$ & $0.0373(3)$ & $0.1717(3)$ & $0.166(4)$ & $0.058(1)$ & $0.080(1)$ & $-0.015(2)$ & $0.016(2)$ & $-0.0179(9)$ \\
\hline$N(1)$ & $2 i$ & & $0.4235(3)$ & $0.3205(3)$ & $0.4321(2)$ & $0.053(2)$ & $0.053(2)$ & $0.045(2)$ & $-0.011(1)$ & $-0.013(1)$ & $-0.011(2)$ \\
\hline $\mathrm{N}(2)$ & $2 i$ & & $0.3033(3)$ & $0.5368(2)$ & $0.3540(2)$ & $0.055(2)$ & $0.053(2)$ & $0.049(2)$ & $-0.013(1)$ & $-0.018(1)$ & $-0.011(1)$ \\
\hline $\mathbf{N}(3)$ & $2 i$ & & $0.3698(3)$ & $0.4884(2)$ & $0.1535(2)$ & $0.059(2)$ & $0.051(2)$ & $0.036(2)$ & $-0.021(1)$ & $-0.004(1)$ & $-0.010(1)$ \\
\hline $\mathbf{N}(4)$ & $2 i$ & & $0.2471(3)$ & $0.3222(2)$ & $0.2875(2)$ & $0.057(2)$ & $0.047(2)$ & $0.035(2)$ & $-0.017(1)$ & $-0.003(1)$ & $-0.006(1)$ \\
\hline $\mathbf{N}(5)$ & $2 i$ & & $0.5987(3)$ & $0.4755(3)$ & $0.2587(2)$ & $0.063(2)$ & $0.078(2)$ & $0.062(2)$ & $-0.023(2)$ & $-0.012(2)$ & $-0.017(2)$ \\
\hline $\mathbf{N}(6)$ & $2 i$ & & $0.5415(3)$ & $0.2473(3)$ & $0.2419(2)$ & $0.067(2)$ & $0.065(2)$ & $0.054(2)$ & $-0.015(2)$ & $-0.006(2)$ & $-0.013(2)$ \\
\hline$C(1)$ & $2 i$ & & $0.2438(4)$ & $0.6097(3)$ & $0.5046(3)$ & $0.088(3)$ & $0.084(3)$ & $0.064(3)$ & $-0.001(2)$ & $-0.017(2)$ & $-0.039(2)$ \\
\hline$C(2)$ & $2 i$ & & $0.2992(3)$ & $0.5168(3)$ & $0.4451(3)$ & $0.052(2)$ & $0.063(3)$ & $0.051(2)$ & $-0.012(2)$ & $-0.015(2)$ & $-0.019(2)$ \\
\hline C(3) & $2 i$ & & $0.3535(3)$ & $0.3917(3)$ & $0.4929(2)$ & $0.049(2)$ & $0.064(2)$ & $0.045(2)$ & $-0.015(2)$ & $-0.014(2)$ & $-0.015(2)$ \\
\hline C(4) & $2 i$ & & $0.3254(4)$ & $0.3494(4)$ & $0.5925(3)$ & $0.076(3)$ & $0.076(3)$ & $0.044(2)$ & $-0.017(2)$ & $-0.016(2)$ & $-0.016(2)$ \\
\hline$C(5)$ & $2 i$ & & $0.3688(4)$ & $0.2293(4)$ & $0.6304(3)$ & $0.086(3)$ & $0.089(3)$ & $0.047(2)$ & $-0.021(2)$ & $-0.018(2)$ & $-0.007(2)$ \\
\hline$C(6)$ & $2 i$ & & $0.4399(4)$ & $0.1559(4)$ & $0.5706(3)$ & $0.081(3)$ & $0.061(3)$ & $0.060(3)$ & $-0.016(2)$ & $-0.026(2)$ & $0.007(2)$ \\
\hline $\mathrm{C}(7)$ & $2 i$ & & $0.4667(4)$ & $0.2045(3)$ & $0.4716(3)$ & $0.063(2)$ & $0.060(3)$ & $0.059(2)$ & $-0.009(2)$ & $-0.014(2)$ & $-0.011(2)$ \\
\hline $\mathrm{C}(8)$ & $2 i$ & & $0.2486(4)$ & $0.6533(3)$ & $0.3000(3)$ & $0.069(3)$ & $0.057(3)$ & $0.053(2)$ & $-0.009(2)$ & $-0.017(2)$ & $-0.021(2)$ \\
\hline $\mathrm{C}(9)$ & $2 i$ & & $0.3264(5)$ & $0.7461(4)$ & $0.2676(3)$ & $0.091(3)$ & $0.069(3)$ & $0.079(3)$ & $-0.023(2)$ & $-0.037(3)$ & $-0.011(2)$ \\
\hline$C(10)$ & $2 i$ & & $0.2782(5)$ & $0.8529(4)$ & $0.2078(3)$ & $0.124(4)$ & $0.058(3)$ & $0.091(3)$ & $-0.025(3)$ & $-0.032(3)$ & $-0.012(3)$ \\
\hline$C(11)$ & $2 i$ & & $0.1518(6)$ & $0.8688(4)$ & $0.1792(3)$ & $0.121(4)$ & $0.068(3)$ & $0.070(3)$ & $0.004(3)$ & $-0.039(3)$ & $-0.019(2)$ \\
\hline$C(12)$ & $2 i$ & & $0.0775(5)$ & $0.7766(4)$ & $0.2111(3)$ & $0.089(3)$ & $0.083(3)$ & $0.076(3)$ & $0.003(3)$ & $-0.035(3)$ & $-0.021(3)$ \\
\hline$C(13)$ & $2 i$ & & $0.1228(4)$ & $0.6687(4)$ & $0.2717(3)$ & $0.071(3)$ & $0.069(3)$ & $0.066(3)$ & $-0.012(2)$ & $-0.020(2)$ & $-0.021(2)$ \\
\hline$C(14)$ & $2 i$ & & $0.1031(6)$ & $0.9862(4)$ & $0.1098(4)$ & $0.195(6)$ & $0.082(4)$ & $0.107(4)$ & $0.017(4)$ & $-0.066(4)$ & $-0.004(3)$ \\
\hline$C(15)$ & $2 i$ & & $0.0986(5)$ & $0.2952(4)$ & $0.1856(3)$ & $0.120(4)$ & $0.107(4)$ & $0.079(3)$ & $-0.069(3)$ & $-0.042(3)$ & $0.007(3)$ \\
\hline$C(16)$ & $2 i$ & & $0.2048(4)$ & $0.3516(3)$ & $0.2082(2)$ & $0.059(2)$ & $0.058(2)$ & $0.048(2)$ & $-0.022(2)$ & $-0.010(2)$ & $-0.013(2)$ \\
\hline$C(17)$ & $2 i$ & & $0.2625(4)$ & $0.4542(3)$ & $0.1337(2)$ & $0.066(2)$ & $0.054(2)$ & $0.035(2)$ & $-0.016(2)$ & $-0.006(2)$ & $-0.011(2)$ \\
\hline$C(18)$ & $2 i$ & & $0.2106(4)$ & $0.5123(4)$ & $0.0510(3)$ & $0.078(3)$ & $0.085(3)$ & $0.049(2)$ & $-0.027(2)$ & $-0.020(2)$ & $-0.008(2)$ \\
\hline$C(19)$ & $2 i$ & & $0.2659(4)$ & $0.6111(4)$ & $-0.0101(3)$ & $0.087(3)$ & $0.083(3)$ & $0.041(2)$ & $-0.019(2)$ & $-0.018(2)$ & $0.004(2)$ \\
\hline$C(20)$ & $2 i$ & & $0.3719(4)$ & $0.6479(3)$ & $0.0117(3)$ & $0.090(3)$ & $0.064(3)$ & $0.039(2)$ & $-0.023(2)$ & $-0.007(2)$ & $0.002(2)$ \\
\hline $\mathrm{C}(21)$ & $2 i$ & & $0.4218(4)$ & $0.5845(3)$ & $0.0927(2)$ & $0.069(2)$ & $0.056(2)$ & $0.045(2)$ & $-0.025(2)$ & $-0.003(2)$ & $-0.009(2)$ \\
\hline $\mathrm{C}(22)$ & $2 i$ & & $0.2052(3)$ & $0.2223(3)$ & $0.3651(2)$ & $0.051(2)$ & $0.055(2)$ & $0.048(2)$ & $-0.022(2)$ & $-0.005(2)$ & $-0.010(2)$ \\
\hline$C(23)$ & $2 i$ & & $0.1289(3)$ & $0.2438(3)$ & $0.4532(2)$ & $0.053(2)$ & $0.060(2)$ & $0.053(2)$ & $-0.019(2)$ & $-0.003(2)$ & $-0.012(2)$ \\
\hline$C(24)$ & $2 i$ & & $0.1028(4)$ & $0.1493(4)$ & $0.5324(3)$ & $0.062(2)$ & $0.083(3)$ & $0.050(2)$ & $-0.029(2)$ & $-0.001(2)$ & $-0.008(2)$ \\
\hline$C(25)$ & $2 i$ & & $0.1490(4)$ & $0.0309(4)$ & $0.5261(3)$ & $0.060(3)$ & $0.069(3)$ & $0.064(3)$ & $-0.029(2)$ & $-0.015(2)$ & $0.003(2)$ \\
\hline$C(26)$ & $2 i$ & & $0.2231(4)$ & $0.0092(3)$ & $0.4373(3)$ & $0.071(3)$ & $0.054(2)$ & $0.080(3)$ & $-0.021(2)$ & $-0.014(2)$ & $-0.006(2)$ \\
\hline$C(27)$ & $2 i$ & & $0.2512(4)$ & $0.1041(3)$ & $0.3572(3)$ & $0.065(2)$ & $0.060(3)$ & $0.059(2)$ & $-0.021(2)$ & $-0.002(2)$ & $-0.016(2)$ \\
\hline$C(28)$ & $2 i$ & & $0.1247(4)$ & $-0.0725(4)$ & $0.6145(3)$ & $0.089(3)$ & $0.101(3)$ & $0.077(3)$ & $-0.041(3)$ & $-0.021(3)$ & $0.028(3)$ \\
\hline$C(29)$ & $2 i$ & & $0.6955(4)$ & $0.5215(4)$ & $0.2205(3)$ & $0.067(3)$ & $0.097(3)$ & $0.056(3)$ & $-0.030(2)$ & $-0.007(2)$ & $-0.038(2)$ \\
\hline$C(30)$ & $2 i$ & & $0.5891(4)$ & $0.1620(4)$ & $0.2140(3)$ & $0.095(3)$ & $0.055(3)$ & $0.040(2)$ & $-0.008(2)$ & $0.000(2)$ & $0.000(2)$ \\
\hline
\end{tabular}

Acknowledgment. The author is thankful for financial support from research project of the Education Department of Shaanxi Province (grant no. 05JK136).

\section{References}

1. El-Masry, A. H.; Fahmy, H. H.; Abdelwahed, S. H. A.: Synthesis and antimicrobial activity of some new benzimidazole derivatives. Molecules 5 (2000) 1429-1438.

2. Zhong, X.; Yi, J.; Sun, J.; Wei, H. L.; Liu, W. S.; Yu, K. B.: Synthesis and crystal structure of some transition metal complexes with a novel bisSchiff base ligand and their antitumor activities. Europ. J. Med. Chem. 41 (2006) 1090-1092.

3. Liang, H.; Wang, X. J.; Cong, Y. L.; Li, S. T.; Zeng, J. Q.: Synthesis and Crystal Structure of Schiff Base $N, N$ '-Bis(2-Hydroxylbenzylidene)-2Hydroxyl-1,3-propanediamine. Chinese J. Struct. Chem. 16 (1997) 141143.
4. Wang, G. Q.; Cai, J.; Zhang, Y. Q.; Zhang, Q. J.: Synthesis and crystal structure of a novel diketone Schiff base. Chinese J. Appl. Chem. 26 (2009) 329-332

5. Li, J. F.: The synthesis and the characterization of the two new derivatives of luteolin. J. Nat. Sci. Heilongjiang Uni. 26 (2009) 255-257.

6. He, Q. L.; Li, J.: Coordination number and coordination structure of metal complex. Nonferrous Metals 58 (2006) 73-76.

7. Yang, X. G.; Zhao, Q. L.; Xuan, Z. W.: Synthesis and spectral properties of a novel Naphthalimide/Pyrazolone Schiff base with solvatochromism and fluorescence proton switches. J. Hunan Uni. 35 (2008) 58-63.

8. Sheldrick, G. M.: SHELXS-97. Program for the Solution of Crystal Structures. University of Göttingen, Germany 1997.

9. Sheldrick, G. M.: SHELXL-97. Program for the Refinement of Crystal Structures. University of Göttingen, Germany 1997.

10. Sheldrick, G. M.: SHELXTL. Structure Determination Software Suite. Version 6.14. Bruker AXS, Madison, Wisconsin, USA 2000. 